\title{
Chest imaging for COVID-19: a single-center comparative results in pediatric patients
}

\author{
H. Nursun Özcan ${ }^{1 \oplus}$, Yasemin Özsürekci $^{2 \oplus}$, Berna Oğuz ${ }^{1 \oplus}$, Pembe Derin Oygar $^{2 \oplus}$, \\ Sibel Laçinel Gürlevik ${ }^{2 \oplus}$, Jale Karakaya ${ }^{3 \oplus}$, Özlem Tekşam ${ }^{4 \oplus}$, Mehmet Ceyhan ${ }^{2 \oplus,}$ \\ Mithat Haliloğlu ${ }^{1 \oplus}$ \\ Departments of ${ }^{1}$ Pediatric Radiology, ${ }^{2}$ Pediatric Infectious Diseases, ${ }^{3}$ Biostatistics and ${ }^{4}$ Pediatric Emergency, Hacettepe University \\ Faculty of Medicine Ankara, Turkey.
}

\begin{abstract}
Background. Chest computed tomography (CT) appears to be an important radiological modality for the diagnosis of COVID-19 in adults. Studies comparing the findings of such children with those of other viral infections have not been reported either. The aim of this study was to present comparative imaging findings of 75 pediatric COVID-19 patients and four patients with other viral upper respiratory tract infections. We also aimed to demonstrate the possible association between the radiological and laboratory findings in the COVID group.
\end{abstract}

Methods. From 11 March 2020 to 20 June 2020, 79 children (aged < 18 years) were enrolled. COVID-19 was detected by RT-PCR or antibody testing. A plain chest X-ray was obtained from all subjects. Non-contrast chest CT was performed for symptomatic patients.

Results. Seventy-five patients had COVID-19 and 4 were infected with other pathogens i.e. adenovirus, rhinovirus, parainfluenza virus $B$, respiratory syncytial virus. The ages of the patients $(36 \mathrm{M}, 43 \mathrm{~F})$ ranged from 7 months to 17 years old. The sensitivity of chest X-ray (as compared to RT-PCR) was 10.67\% (95 CI\%: $4.72-19.94 \%)$. From 23 chest CT's five of them were normal and nine of them had only nodules $(<5 \mathrm{~mm})$. The sensitivity of CT was $78.26 \%$ (95CI\%: $54.30-92.54 \%$ ), false-negative rate was $21.7 \%$.

Conclusions. The sensitivity of chest CT was found to be low and any significant correlations could have not been depicted, between the radiological parameters and the presence of lymphopenia. Clinical follow-up combined with corresponding pathogen detection, and chest CT of the symptomatic COVID-19 patients might be a feasible/prompt protocol in children.

Key words: SARS-COV-2, children, radiology, CT, X-ray.

An outbreak of 2019 novel coronavirus (COVID-19) infection was first discovered in Wuhan City, China - turning into a global health pandemic thereafter. ${ }^{1,2}$ Likewise, the number of patients is rapidly increasing out of China; and especially Europe and America are currently severely affected.

\footnotetext{
$凶$ H. Nursun Özcan

drhnozcan@yahoo.com
}

Received 14th December 2020, revised 2nd September 2021, 14th November 2021, accepted 29th November 2021.
In the early days of the COVID-19 infection outbreak, pediatric patients were extremely rare. ${ }^{3}$ Later on, it has been reported that COVID-19 can be spread within the whole age spectrum. ${ }^{4,5}$ One of the recent epidemiologic studies has revealed that children of all ages appeared susceptible to COVID-19. ${ }^{6}$ Additionally, newborns of COVID-19 infected mothers have also increased the concern further. ${ }^{7}$

Upper respiratory tractinfections due to different viral etiologies are common in the pediatric age group. Additionally, the total positive rate of 
reverse transcription polymerase chain reaction (RT-PCR) for nasopharyngeal swab samples was reported to be $30-60 \%$ at the initial presentation of COVID-19. ${ }^{8}$ Distinguishing COVID-19 from other common respiratory tract infections in the pediatric population is a big dilemma. In adults, chest computed tomography (CT) appears to be an important radiological modality for the diagnosis of COVID-19 showing typical imaging features like ground-glass opacities, multifocal patchy consolidation, and/or interstitial changes with a peripheral distribution. ${ }^{9}$ Disease severity in COVID-19 is associated with blood cell alterations, C-Reactive Protein levels and inflammatory markers. The relationship between radiological and laboratory findings were displayed in a few studies in adult patients. ${ }^{10,11}$ To this end, however, there is limited data reported for pediatric COVID-19 patients. Further, to the best of our knowledge, studies comparing the findings of such children with those of other viral infections have not been reported either. Accordingly, the purpose of this study was two-fold. First, we aimed to present comparative imaging findings of 75 pediatric COVID-19 patients and four patients with other viral upper respiratory tract infections. Second, we also aimed to demonstrate the possible association between the radiological and laboratory findings in the COVID group.

\section{Material and Methods}

\section{Subjects and study design}

This was a single-center, retrospective, observational study conducted at a tertiary hospital. From 11 March 2020 to 20 June 2020, a total of 79 children (aged $<18$ years) were enrolled. Written informed consent was obtained from the patients or their parents, and the study protocol was approved by the ethics committees of the Ministry of Health and Hacettepe University (GO 20/392). Demographic data and clinical features as well as laboratory (white blood cell, absolute lymphocyte count, C-reactive protein, procalcitonin) and imaging findings were obtained for each enrolled patient.
Viral respiratory panel tests were performed on all patients, to exclude the superimposed or concomitant pneumonia due to different infectious agents.

\section{Radiological evaluations}

Plain chest X-rays were obtained from all subjects. Non-contrast chest CT was performed for symptomatic patients i.e. having fever, cough, respiratory distress. Only one asymptomatic patient received a chest CT due to strong family positivity (seven people from the family were covid positive). Chest CT studies were performed using the SOMATOM Definiton AS 64 unit (Siemens Medical System; Siemens, Germany) with the following parameters: $80 \mathrm{kV}, 45$ to $70 \mathrm{~mA}, 1.2-$ mm collimation, and 1:5 pitch. The scanning range covered from lung apex to diaphragm on axial plane taken under free breathing with the patients in the supine position. Thin-section CT images were reconstructed with $1.25-\mathrm{mm}$ collimation with a standard algorithm and then sent to the picture archiving and communication system (PACS) for analysis. CT images were evaluated using a lung window with a window level of $-600 \mathrm{HU}$ and window width of 1500 $\mathrm{HU}$, and the soft-tissue window with a window level of $40 \mathrm{HU}$ and window width of 300 HU. All the images were stored in PACS and reviewed by three expert pediatric radiologists (having, 25, 17 and 7 years of background in pediatric radiology) by consensus who were blinded to the symptoms and laboratory tests. The CT features were evaluated for: (a) ground-glass opacities (GGO), (b) nodules, (c) consolidations, (d) crazy paving, (e) atelectasis, (f) peribronchial thickening, (g) tree-in-bud sign, (h) pleural effusion, (i) lymphadenopathy (short axis dimension $>1.0 \mathrm{~cm}$ ) and (j) white lung. The anatomic distribution (unilateral, bilateral, subpleural, central or mixed), zonal predominance (upper, middle, lower lung; central, middle, or peripheral location), and extent (focal, multifocal, and diffuse) of the lesions were also recorded. Visual quantitative assessment score (VQAS) was performed 
according to the extent of opacities (including GGOs, crazy paving and consolidation) in each lobe. Scores were defined as following: 0 (none), 1 (affecting $<5 \%$ of the lobe), 2 (affecting $5 \%-25 \%$ of the lobe), 3 (affecting $26 \%-49 \%$ of the lobe), 4 (affecting $50 \%-75 \%$ of the lobe) and 5 (affecting $>75 \%$ of the lobe). A maximum CT score of 5 was possible for each lobe. Total CT score was reached by summing the scores in five lobes (range from 0 to 25 ). ${ }^{12}$

\section{COVID-19 diagnosis}

COVID-19 was detected by RT-PCR obtained from the nasopharyngeal swab and/or tracheal aspirate specimens or antibody testing (Ig $\mathrm{M}$ and $\mathrm{Ig}$ G) via COVID-19 [SARS-CoV-2] Antibody Test Kit; Colloidal Gold. ${ }^{13}$ Multiplex Real time PCR (RT-PCR) method was used for the detection of other respiratory viral agents, as well. Nucleic acid isolation was performed by Gene All Ribospin vRD II Isolation Kit, Seoul, Korea and RT- PCR method was carried out by Seegene RV16 Detection Kit, Seoul, Korea.

\section{Disease severity}

We categorized the severity of cases based on the clinical characteristics, laboratory results and imaging findings as described elsewhere: ${ }^{6}$ a) asymptomatic infection; no clinical and/or radiological findings, b) mild disease; acute upper respiratory tract infection without clinical and radiological pneumonia, c) moderate disease; pneumonia with the symptoms of respiratory tract infection, d) severe disease; progressive respiratory disease with dyspnea and central cyanosis, e) critically ill cases; acute respiratory distress syndrome or respiratory failure, shock, organ dysfunction including encephalopathy, myocardial injury, coagulation abnormalities, and acute kidney injury.

\section{Statistical analysis}

IBM SPSS software package version 23.0 was used for all the statistical analyses. Categorical variables are expressed as frequencies and percentages. Mean \pm standart deviation was given as descriptive statistics for the numeric variables. Fisher exact test was used to compare the distribution of a categorical variable. In order to evaluate the accuracy of the diagnosis, sensitivity and its confidence intervals (CI) were obtained as a test performance measures of CT. ${ }^{14}$ The sensitivity value of CT obtained for children from this study was compared with the sensitivity value obtained from a previously published study in adults. ${ }^{15}$ Two sample proportion test was used to determine whether the proportions of two independent groups differ. The results of X-ray and CT were compared with McNemar test. The relationship between lymphopenia and RT-PCR positivity were examined with the "Yates (Continuity Correction) Chi square" test.

\section{Results}

Demographic and clinical characteristics of patients with COVID-19 are given in Table I. Ages of the patients ( $36 \mathrm{M}, 43 \mathrm{~F}$ ) ranged from 7 months to 17 years with a mean value of 10.5 years (SD 5.2). Overall; 75 patients had COVID-19 and 4 were infected with only other pathogens i.e. adenovirus, rhinovirus, parainfluenza virus B and respiratory syncytial virus (RSV). All four patients with other infections had a chest CT, as they suffered from similar symptoms and/or suspicion pertaining to COVID-19. Of the 75 COVID-19 patients 67 received the diagnosis with RT-PCR and eight of them had positive antibody tests.

Among the COVID-19 patients, 23 had a chest $\mathrm{CT}$ and plain X-ray while the remaining $52 \mathrm{had}$ only a chest X-ray whereby 23 of them were asymptomatic, 42 had mild, 7 had moderate, and 3 had a critically ill disease course. Since one of the critically ill patients had severe cardiac failure and was later on lost, a CT could not be performed. Extracorporeal membrane oxygenation was used in another critically ill patient with refractory acute respiratory distress syndrome secondary to StevensJohnson syndrome and was later on lost. 


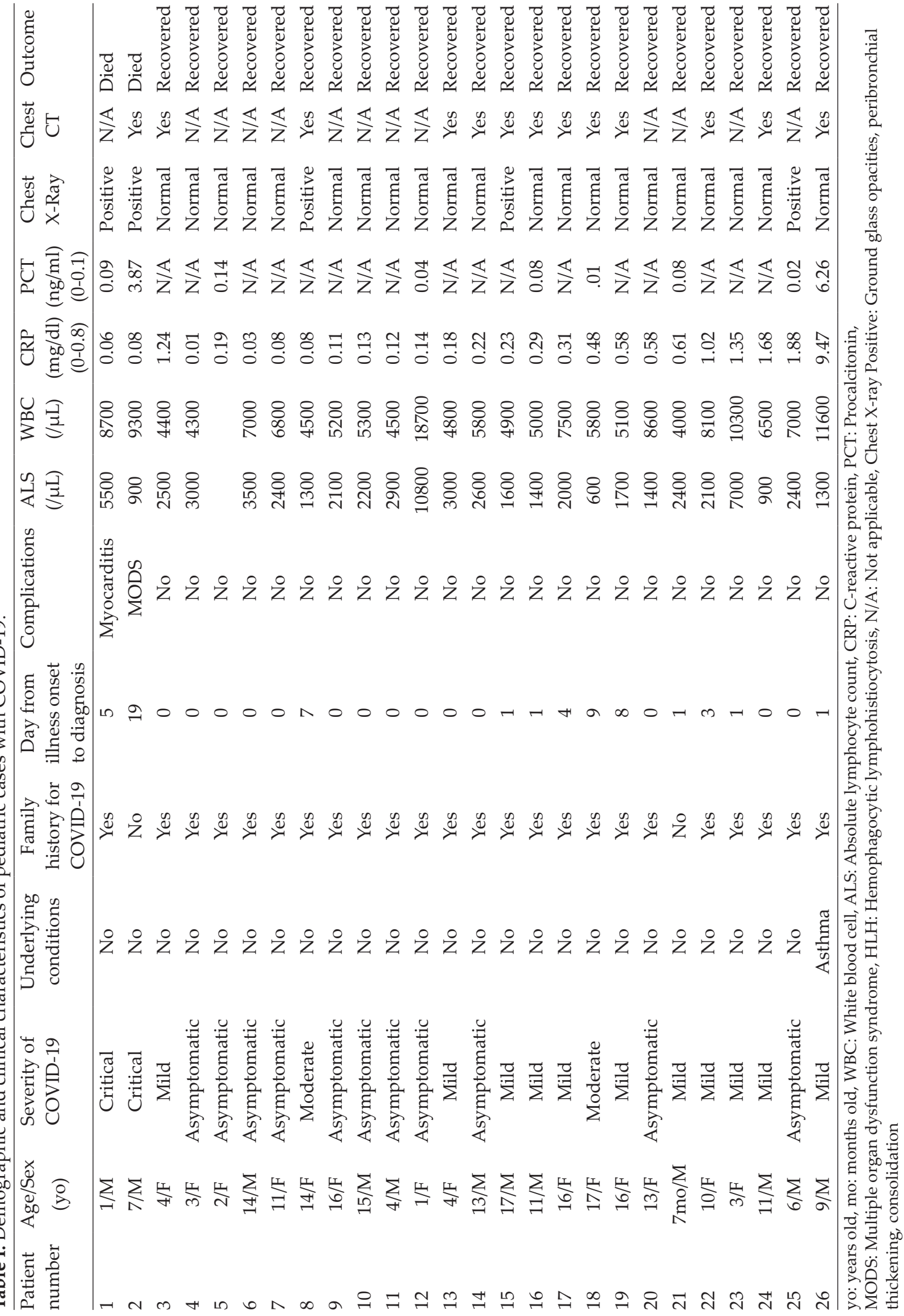




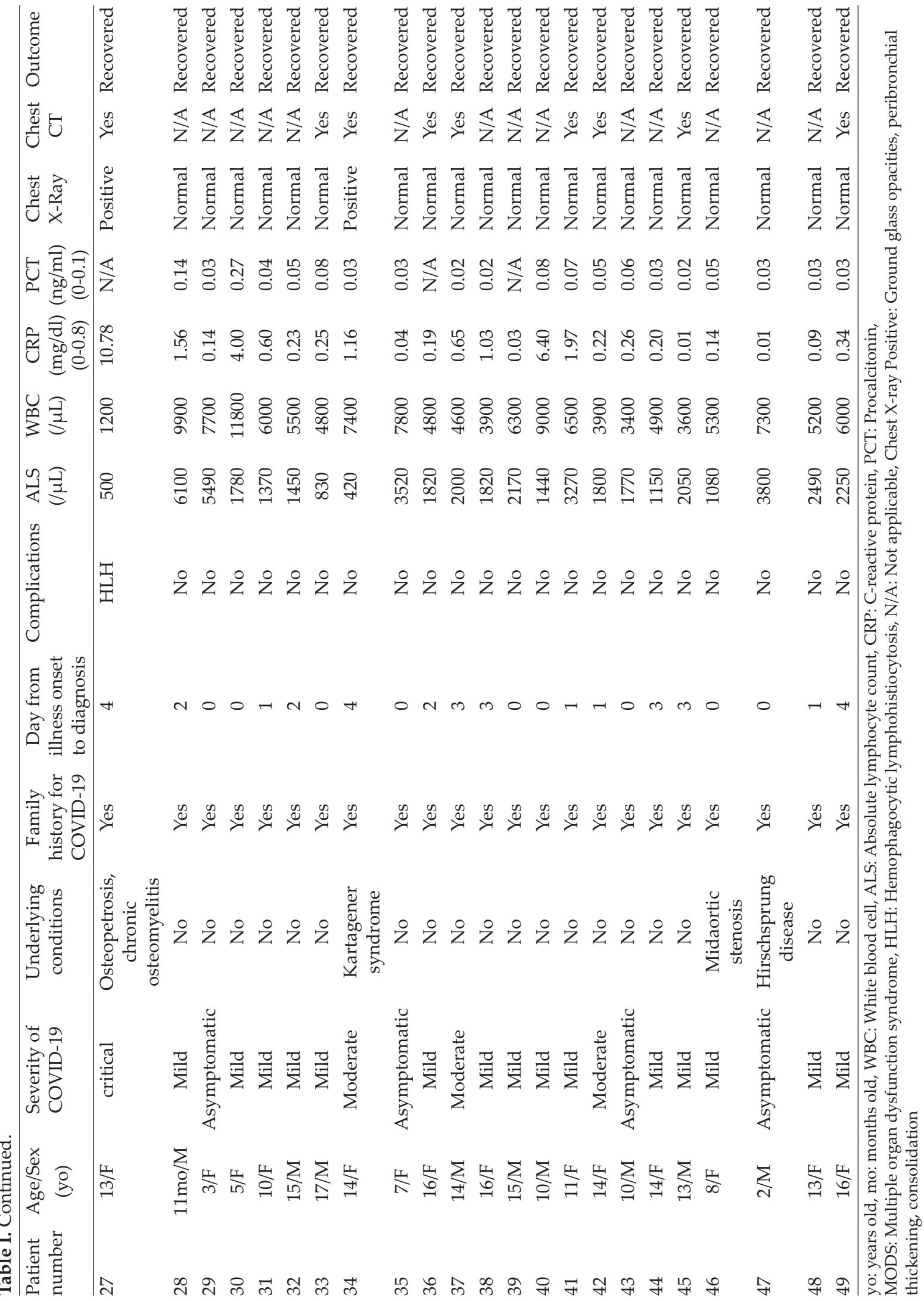




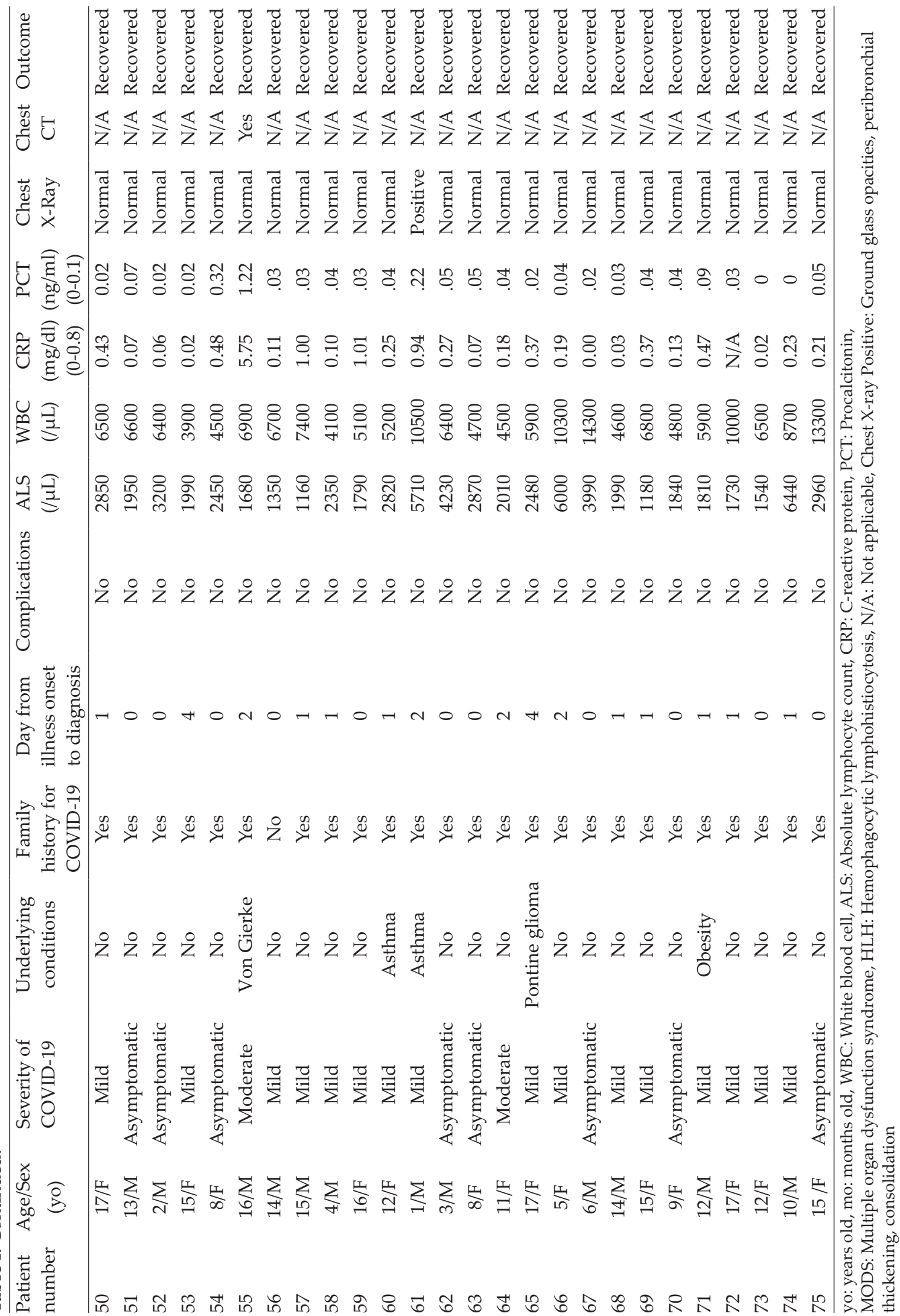


Sixty-seven patients had normal chest X-ray findings. A total of eight children had remarkable abnormalities (GGO, peribronchial thickening, consolidation) on X-ray. The sensitivity of chest X-ray (as compared to RT-PCR) was 10.67\% (95 CI\%: $4.72-19.94 \%)$. More than half of the chest CTs were obtained on the same day with chest X-rays (58\%), while $25 \%$ were obtained the next day. The remaining $16 \%$ percent of chest CTs were obtained between 2-6 days.

The CT findings of pediatric patients with COVID-19 infection are given in Table II.
Visual quantitative assessment score (VQAS) and the comparison with the clinical severity of the COVID-19 patient are given in Table III. From 23 chest CT's five of them were normal and nine of them had only subpleural and/ or intraparachymal nodules $(<5 \mathrm{~mm})$. The sensitivity of the CT (as compared to RT-PCR) was $78.26 \%$ (95CI\%: $54.30-92.54 \%$ ), the false negative rate was $21.7 \%$. The most frequent chest CT findings were nodules, GGO, GGO and consolidation and peribronchial thickening (Fig. 1 and Fig. 2). Of note, most of the patients had bilateral chest $\mathrm{CT}$ findings.

Table II. Computed tomography findings of patients with COVID-19 (N=23).

\begin{tabular}{|c|c|c|c|c|}
\hline \multirow{2}{*}{ Findings } & \multicolumn{4}{|c|}{ Asymptomatic $(n=1)$ Mild $(n=13)$ Moderate $(n=7)$ Critically ill $(n=2)$} \\
\hline & $\mathrm{N}(\%)$ & $\mathrm{N}(\%)$ & $\mathrm{N}(\%)$ & $\mathrm{N}(\%)$ \\
\hline Ground glass opacities & $1(4.54)$ & - & $7(30.4)$ & $1(4.54)$ \\
\hline Nodule & $1(4.54)$ & $7(31.8)$ & $5(22.7)$ & - \\
\hline Consolidation & - & - & - & - \\
\hline Crazy Paving & - & - & - & $1(4.54)$ \\
\hline Atelectasis & - & - & - & - \\
\hline Ground glass opacities and consolidation & - & - & $1(4.54)$ & $2(9.09)$ \\
\hline Peribronchial thickening & - & - & $4(18.1)$ & $1(4.54)$ \\
\hline Tree in buds & - & - & - & - \\
\hline Pleural effusion & - & - & - & - \\
\hline \multicolumn{5}{|l|}{ Bronchiectasis } \\
\hline Lymhadenopathy & - & - & - & - \\
\hline White lung & - & - & - & $1(4.54)$ \\
\hline \multicolumn{5}{|l|}{ Lung region distribution } \\
\hline Unilateral & - & $2(9.09)$ & $1(4.54)$ & - \\
\hline Bilateral & $1(4.54)$ & $5(22.7)$ & $5(22.7)$ & $2(9.09)$ \\
\hline Subpleural & $1(4.54)$ & $8(36.3)$ & $5(22.7)$ & $1(4.54)$ \\
\hline Central & - & - & - & - \\
\hline Mixed & - & - & $4(18.1)$ & $1(4.54)$ \\
\hline \multicolumn{5}{|l|}{ Lung lobe involved } \\
\hline Right upper lobe & $1(4.54)$ & $4(18.1)$ & $3(13.6)$ & $2(9.09)$ \\
\hline Right middle lobe & $1(4.54)$ & $3(13.6)$ & $2(9.09)$ & $1(4.54)$ \\
\hline Right lower lobe & $1(4.54)$ & $5(22.7)$ & $5(22.7)$ & $2(9.09)$ \\
\hline Left upper lobe & $1(4.54)$ & $3(13.6)$ & $4(18.1)$ & $1(4.54)$ \\
\hline Left lower lobe & - & $6(27.2)$ & $6(27.2)$ & $2(9.09)$ \\
\hline \multicolumn{5}{|l|}{ Distribution } \\
\hline Focal & - & $1(4.54)$ & $1(4.54)$ & - \\
\hline Multifocal & $1(4.54)$ & - & $5(22.7)$ & $1(4.54)$ \\
\hline Diffuse & - & - & - & $1(4.54)$ \\
\hline Normal CT & - & $5(22.7)$ & - & - \\
\hline
\end{tabular}


Table III. Visually calculated total CT scores of the pulmonary involvement in 23 COVID-19 patients.

\begin{tabular}{|c|c|c|c|c|c|c|c|c|c|}
\hline Clinical severity & & & & lcul & & $\mathrm{SCO}$ & & & \\
\hline & 0 & 1 & 2 & 3 & 4 & 5 & 7 & 10 & 25 \\
\hline Asymptomatic $(\mathrm{n}=1)$ & & & & 1 & & & & & \\
\hline Mild (n=13) & 5 & 2 & 2 & 2 & 1 & 1 & & & \\
\hline Moderate $(\mathrm{n}=7)$ & & 1 & 1 & & & 1 & 1 & 3 & \\
\hline Critical (n=2) & & & & & & & & & 2 \\
\hline
\end{tabular}

n: Number of patients

Significant positive correlations were found between disease severity and most common CT findings of COVID-19 (peripheral, subpleural and perilymphatic GGO $\quad(p=0.001)$. One asymptomatic patient had peripheral GGO. The most common CT findings of COVID-19 were

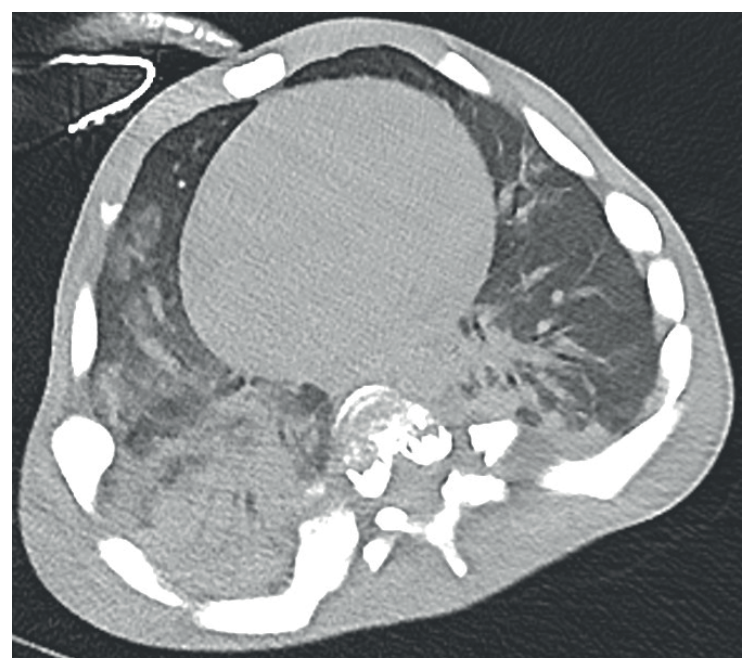

Fig. 1. A-13-year-old girl with COVID-19 pneumonia. Axial non-contrast enhanced chest CT image demonstrates diffuse consolidation in the right lower lobe, peribronchial patchy ground-glass opacities in the right middle lobe, and subpleural consolidation in the left lower lobe. Note that the diffuse osteosclerosis of all bony structures in the thoracic cage is compatible with osteopetrosis. present in $7.7 \%$ of the mild group, $71.4 \%$ of the moderate group and $100 \%$ of the critical ill group. The sensitivity rate of the most common CT findings of COVID-19 (peripheral, subpleural and perilymphatic GGO) as compared to RTPCR was 39.13\% (95CI\%: $19.71-61.46 \%$ ). The time interval from symptom onset to chest CT was between 2-9 days (median 2.5 days). Chest $\mathrm{X}$-ray and the most common $\mathrm{CT}$ findings of COVID-19 were similar ( $\mathrm{p}=0.453)$.

Five patients had a normal chest $\mathrm{CT}$, and they were asymptomatic or had a mild disease course as well. Statistically significant positive correlations were found between disease severity and lymphopenia $(p=0.009)$. Lymphopenia was present in $4.3 \%$ of the asymptomatic group, $28.6 \%$ of the mild group, $42.9 \%$ of the moderate and $66.7 \%$ of the critically ill group. No significant correlations were depicted between the radiological parameters and lymphopenia. Yet, $77.8 \%$ of patients with lymphopenia and $78.6 \%$ of those without had positive CT findings. No significant correlations were depicted between RT-PCR positivity and lymphopenia $(\mathrm{p}=0.671)$.

Table IV demonstrates the imaging features of patients with other infections. These

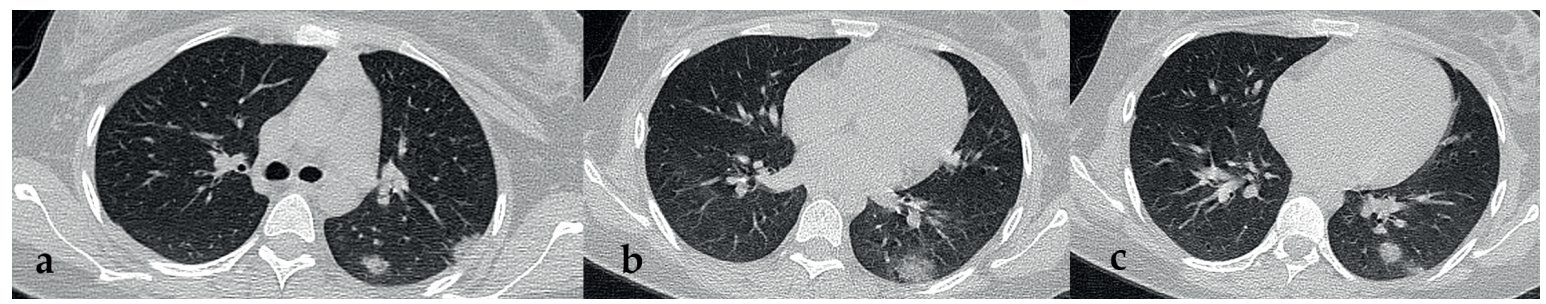

Fig. 2a-c. A-17-year-old girl with COVID-19 pneumonia. (a-c) Axial non-contrast enhanced chest CT images demonstrate subpleural ground-glass nodules in the left lower lobe. 
Table IV. Computed tomography findings of other viral infections.

\begin{tabular}{|c|c|c|c|c|}
\hline Findings & Adenovirus & Rhinovirus & RSV B & RSV A/Parainfluenza \\
\hline Ground glass opacities & + & + & + & - \\
\hline Nodule & + & - & - & - \\
\hline Consolidation & + & - & + & + \\
\hline Crazy paving & - & - & - & - \\
\hline Atelectasis & - & + & + & - \\
\hline Ground glass opacities and consolidation & + & - & - & + \\
\hline Peribroncial thickening & - & + & + & - \\
\hline Tree in buds & - & - & - & - \\
\hline Pleural effusion & - & - & - & + \\
\hline Bronchiectasis & + & - & - & - \\
\hline Lympadenopathy & - & - & - & - \\
\hline White lung & - & - & - & - \\
\hline \multicolumn{5}{|l|}{ Lung region distribution } \\
\hline Unilateral & - & - & - & - \\
\hline Bilateral & + & + & + & + \\
\hline Subpleural & + & + & + & - \\
\hline Central & + & + & + & + \\
\hline Mixed & + & + & + & + \\
\hline \multicolumn{5}{|l|}{ Lung lobe involved } \\
\hline Right upper lobe & - & - & - & + \\
\hline Right middle lobe & + & + & + & + \\
\hline Right lower lobe & + & + & + & + \\
\hline Left upper lobe & + & + & + & + \\
\hline Left lower lobe & + & + & + & + \\
\hline \multicolumn{5}{|l|}{ Distribution } \\
\hline Focal & - & - & - & - \\
\hline Multifocal & + & + & + & + \\
\hline Diffuse & - & - & - & + \\
\hline Normal CT & - & - & - & - \\
\hline
\end{tabular}

RSV: Respiratory syncytial virus

four patients were infected with other viral respiratory viruses and RT-PCR test was negative for COVID-19. The patient with an adenovirus infection was a 10-year-old boy, who had a fever and cough, his father had Covid-19 positivity. The Rhinovirus infected patient was a 12-year-old boy and had respiratory distress. A 1-year-old girl with Respiratory syncytial virus had a fever and cough. An 8-years-old boy with Respiratory syncytial virus A/Parainfluenza had a fever. Their chest CT imaging findings were similar to those of COVID-19 patients with regards to bilateral involvement and the aforementioned most commonly seen patterns (Fig. 3 and 4).

\section{Discussion}

In this comparative imaging study, we have shown that CT findings of COVID 19 may be diverse in children. Chest CT sensitivity was found to be moderate low (78.26\%) and any significant correlations could have not been depicted between the radiological parameters and the presence of lymphopenia. When compared to the relevant literature whereby 


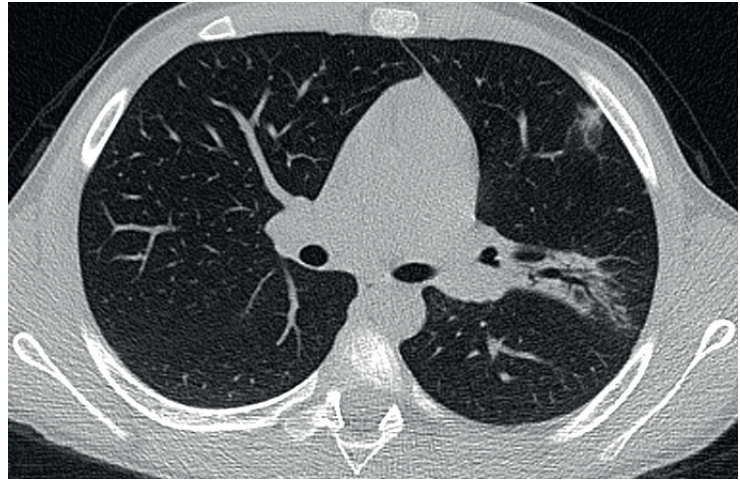

Fig. 3. A-10-year-old boy with adenovirus pneumonia. Axial non-contrast enhanced chest CT image shows subpleural ground-glass nodule in the left upper lobe with consolidation and mild bronchiectasis.

mild pediatric patients showed normal findings on chest $\mathrm{CT}^{16,17}$, five of our 23 cases had a normal $\mathrm{CT}$ and nine of them had only subpleural and parenchymal nodules. Again, GGO (the most frequently observed pattern) mainly in the peripheral, subpleural and posterior lungs was the main finding in our patients. Herein, it is noteworthy that other findings like nodules, GGO with consolidation or peribronchial thickening can also be detected in children. Significant positive correlations were found between disease severity and characteristic COVID-19 CT findings. In our study, both crazy paving pattern and consolidation were more common in critically ill patients. In COVID-19 patients, diffuse alveolar damage has been described with interstitial edema, thickening of alveolar walls and proliferation of interstitial fibroblasts. ${ }^{18}$

COVID-19 viral pneumonia is an acute infectious chest disease due to a novel coronavirus. The clinical spectrum of the disease ranges from asymptomatic to critically ill. Although the severity of the disease seems to be milder in pediatric patients as compared with adults, data about various clinical findings of COVID-19, particularly in children are limited. ${ }^{19,20}$ As such, one of the challenging issues for radiologists, as well as clinicians remains to be the availability to distinguish

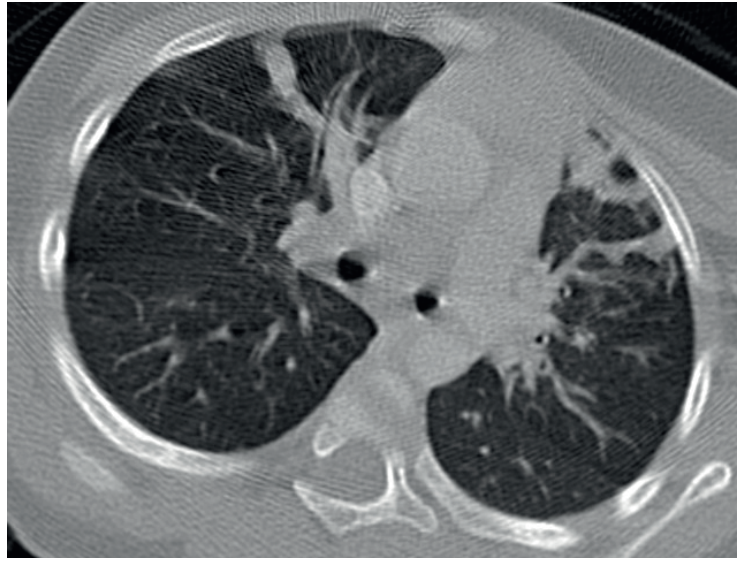

Fig. 4. A-1-year-old girl with respiratory syncytial virus-B infection. Axial non-contrast enhanced chest CT image shows subpleural atelectasis in the upper lobes.

CT findings of children with COVID-19 from those of other viral respiratory infections. Herein, CT findings of bilateral multifocal GGO with patchy consolidations and peribronchial thickening were also present in our patients who had pneumonia due to other infections. On the other hand, the characteristics of pneumonia caused by adenovirus had higher density, more consolidations and fewer subpleural lesions. Concerning both RSV and parainfluenza virus; the characteristics of pneumonia were mostly distributed along the bronchi with bronchial wall thickening. Hence, a definite diagnosis cannot be achieved on the basis of imaging features alone. Indeed, chest CT findings of pneumonia due to different pathogens seem to overlap, and COVID-19 pneumonia can be superimposed with lung involvement due to other pathogens - presenting more severe imaging findings. Therefore, radiological assessment should indisputably be coupled with clinical and laboratory examinations.

In China, chest CT was recommended for the diagnosis at the beginning of the pandemic due to the low positivity of PCR tests as a reflex behavior against a new disease. Moreover, as chest $\mathrm{CT}$ was mentioned to be a more sensitive diagnostic tool rather than RT-PCR, CT has been used for screening/diagnosis for adult patients. ${ }^{15}$ The sensitivity value of CT obtained 
for children from this study was (78.26\%) compared with the sensitivity value obtained from adults $(97 \%)$ a previously published study of Ai et al. ${ }^{15}$ Chest CT had higher sensitivity for COVID-19 diagnosis in adults. Most of the pediatric patients with COVID-19 in the present study had asymptomatic or mild disease course, and their radiological findings were also normal or mild. It is apparent that the sensitivity of chest radiographs (the first examination to be preferred) in showing the GGO and small consolidations is low. Therefore, according to our findings, chest CT should rather be performed for symptomatic pediatric patients. Moreover, this approach would also be crucial to prevent high levels of radiation exposure in children. Steinberger et al. ${ }^{21}$ drew attention to the same issue, and reported that the low incidence of CT exams with positive findings and the low severity of disease in children's CTs should be noted when handling the utility and restrictions of $\mathrm{CT}$ for the assessment of COVID-19 in children and they suggested $\mathrm{CT}$ for evaluating suspected complications of COVID-19.

In a recent study, Wang et al. ${ }^{22}$ reported that the lung abnormalities on CT progressed rapidly after the symptom onset, reached its peak around 6-11 days, and was followed by a lengthy persistence of high levels in adults. A few studies supporting this information have reported an increase in GGOs over time. ${ }^{23}$ Shen et al. ${ }^{13}$ reported that progressive and severe stage of CT findings were rarely seen; however, any association between the clinical and radiological course was not reported. Radiological progression of pediatric cases should not be considered as a hallmark of disease severity and clinical status of the cases should be the mainstay in the management. Again, more case examples and long-term follow-up would be necessary to better demonstrate the development of pediatric COVID-19 disease.

There are some limitations of our study. First, the number of patients infected with other viruses was small. Second, studies with a larger sample size and longer follow-up are awaited to better understand the actual disease course of pediatric COVID-19 patients during management. Lastly, we couldn't set any exclusion criteria such as preexisting diseases may mimic COVID-19 related findings, since the number of patients is small and each patient is very valuable.

In conclusion, pneumonia due to COVID-19 is generally mild in children, and chest CT can well be unremarkable. If present, characteristic imaging findings of COVID-19 infection seem to be subpleural ground-glass opacities and peribronchial thickening. The sensitivity of chest CT was found to be low and any significant correlations could have not been depicted between the radiological parameters and the presence of lymphopenia. However, it would be insufficient to diagnose COVID-19 pneumonia only by CT imaging, and it would actually also be difficult as far as its differential diagnosis from other viral infections is concerned. Therefore, clinical follow-up together with detection of the corresponding pathogens, and chest $\mathrm{CT}$ of the symptomatic patients might be a feasible/prompt protocol in children.

\section{Ethical approval}

Written informed consent was obtained from the patients or their parents, and the study protocol was approved by the ethics committees of the Ministry of Health and Hacettepe University (GO 20/392).

\section{Author contribution}

The authors confirm contribution to the paper as follows: study conception and design: HNÖ; data collection: YÖ, PDO, SLG; analysis and interpretation of results: $\mathrm{BO}, \mathrm{JK}$, ÖT; draft manuscript preparation: HNÖ, MC, MH. All authors reviewed the results and approved the final version of the manuscript. 


\section{Source of funding}

The authors declare the study received no funding.

\section{Conflict of interest}

The authors declare that there is no conflict of interest.

\section{REFERENCES}

1. Zhu N, Zhang D, Wang W, et al. A novel coronavirus from patients with pneumonia in China, 2019. N Eng J Med 2020; 382: 727-733. https://doi.org/10.1056/ NEJMoa2001017

2. World Health Organization. Statement on the second meeting of the International Health Regulations (2005) Emergency Committee regarding the outbreak of novel coronavirus (2019-nCoV). Geneva, Switzerland: WHO, 2020. Available at: https://www.who.int/news/ item/30-01-2020-statement-on-the-secondmeeting-of-the-international-health-regulations(2005)-emergency-committee-regarding-theoutbreak-of-novel-coronavirus-(2019-ncov)

3. Li $\mathrm{Q}$, Guan $\mathrm{X}, \mathrm{Wu} \mathrm{P}$, et al. Early transmission dynamics in Wuhan, China, of novel coronavirusinfected pneumonia. N Engl J Med 2020; 382: 11991207. https://doi.org/10.1056/NEJMoa2001316

4. Guan WJ, Ni ZY, Hu Y, et al. Clinical characteristics of coronavirus disease 2019 in China. N Engl J Med 2020; 382: 1708-1720. https://doi.org/10.1056/ NEJMoa2002032

5. Wu Z, McGoogan JM. Characteristics of and important lessons from the coronavirus disease 2019 (COVID-19) outbreak in China: summary of a report of 72314 cases from the Chinese Center for Disease Control and Prevention. JAMA 2020; 323: 1239-1242. https://doi.org/10.1001/jama.2020.2648

6. Dong $\mathrm{Y}, \mathrm{Mo} \mathrm{X}, \mathrm{Hu} \mathrm{Y}$, et al. Epidemiology of COVID-19 among children in China. Pediatrics 2020; 145: e20200702. https://doi.org/10.1542/peds.20200702

7. Chen $\mathrm{L}, \mathrm{Li} \mathrm{Q}$, Zheng D, et al. Clinical characteristics of pregnant women with Covid-19 in Wuhan, China. N Engl J Med 2020; 382: e100. https://doi. org/10.1056/NEJMc2009226
8. Yang Y, Yang M, Shen C, et al. Evaluating the accuracy of different respiratory specimens in the laboratory diagnosis and monitoring the viral shedding of 2019-nCoV infections. medRxiv 20021493 [Preprint]. https://doi.org/10.1101/2020.02.11.20021493

9. Chung $\mathrm{M}$, Bernheim A, Mei $X$, et al. CT imaging features of 2019 novel coronavirus (2019-nCoV). Radiology 2020;295: 202-207. https://doi.org/10.1148/ radiol.2020200230

10. Tan C, Huang $Y$, Shi F, et al. C-reactive protein correlates with computed tomographic findings and predicts severe COVID-19 early. J Med Virol 2020; 92: 856-862. https://doi.org/10.1002/jmv.25871

11. Wu J, Wu X, Zeng $\mathrm{W}$, et al. Chest $\mathrm{CT}$ findings in patients with coronavirus disease 2019 and its relationship with clinical features. Invest Radiol 2020; 55: 257-261. https://doi.org/10.1097/ RLI.0000000000000670

12. Pan $F, Y e T$, Sun $P$, et al. Time course of lung changes at chest $\mathrm{CT}$ during recovery from coronavirus disease 2019 (COVID-19). Radiology 2020; 295: 715721. https://doi.org/10.1148/radiol.2020200370

13. Shen KL, Yang $\mathrm{YH}$, Jiang RM, et al. Updated diagnosis, treatment and prevention of COVID-19 in children: experts' consensus statement (condensed version of the second edition). World J Pediatr 2020; 16: 232-239. https://doi.org/10.1007/s12519-020$00362-4$

14. Karakaya J. Evaluation of binary diagnostic tests accuracy for medical researches. Turk J Biochem 2021；46: 103-113. https://doi.org/10.1515/tjb-20200337

15. Ai T, Yang Z, Hou H, et al. Correlation of chest $\mathrm{CT}$ and RT-PCR testing in coronavirus disease 2019 (COVID-19) in China: a report of 1014 cases. Radiology 2020; 296: E32-E40. https://doi. org/10.1148/radiol.2020200642

16. Xia W, Shao J, Guo Y, Peng X, Li Z, Hu D. Clinical and $\mathrm{CT}$ features in pediatric patients with COVID-19 infection: different points from adults. Pediatr Pulmonol 2020; 55: 1169-1174. https://doi. org/10.1002/ppul.24718

17. Li W, Cui H, Li K, Fang Y, Li S. Chest computed tomography in children with COVID-19 respiratory infection. Pediatr Radiol 2020; 50: 796-799. https:// doi.org/10.1007/s00247-020-04656-7

18. Tian $\mathrm{S}, \mathrm{Hu}$ W, Niu L, Liu $\mathrm{H}, \mathrm{Xu} H$, Xiao SY. Pulmonary pathology of early-phase 2019 novel coronavirus (COVID-19) pneumonia in two patients with lung cancer. J Thorac Oncol 2020; 5: 700-704. https://doi.org/10.1016/j.jtho.2020.02.010 
19. Chang TH, Wu JL, Chang LY. Clinical characteristics and diagnostic challenges of pediatric COVID-19: a systematic review and meta-analysis. J Formos Med Assoc 2020; 119: 982-989. https://doi.org/10.1016/j. jfma.2020.04.007

20. Ong JSM, Tosoni A, Kim Y, Kissoon N, Murthy S. Coronavirus disease 2019 in critically Ill children: a narrative review of the literature. Pediatr Crit Care Med 2020; 21: 662-666. https://doi.org/10.1097/ PCC. 0000000000002376

21. Steinberger $S$, Lin B, Bernheim A, et al. CT features of coronavirus disease (COVID-19) in 30 pediatric patients. AJR Am J Roentgenol 2020; 215: 1303-1311. https://doi.org/10.2214/AJR.20.23145
22. Wang Y, Dong C, Hu Y, et al. Temporal changes of CT findings in 90 patients with COVID-19 pneumonia: a longitudinal study. Radiology 2020; 296: E55-E64. https://doi.org/10.1148/radiol.2020200843

23. Foust AM, Winant AJ, Chu WC, Das KM, Phillips GS, Lee EY. Pediatric SARS, H1N1, MERS, EVALI, and now coronavirus disease (COVID-19) pneumonia: what radiologists need to know. AJR Am J Roentgenol 2020; 215: 736-744. https://doi. org/10.2214/AJR.20.23267 